\title{
An Analysis of Quality Control in Pap Cytology in A Tertiary Care Centre By Using ASC to SIL Ratio
}

\author{
Hemalatha. J*, Deepak Kumar B, Srinivasa Murthy V and Vani BR \\ Dept of Pathology, ESIC MC \& PGIMSR, Rajajinagar, Bangalore, Karnataka India
}

\begin{abstract}
Introduction: Atypical Squamous Cells(ASC) is a common gynaecologic cytologic abnormality, comprising around 5\% of Papanicolaou test results. It reflects a diagnosis of uncertainty and is used as an intra-laboratory \& inter-laboratory comparison tool for quality control purposes. For this purpose, ASC/SIL ratio can be used as a quality control measure. Bethesda system suggests that ASC/SIL ratio for an individual or laboratory should be less than 2:1 or 3:1.
\end{abstract}

Objectives: To assess the ratio of ASC/SIL categories and thereby evaluation of quality control in gynaecologic cytology smears.

Methods: The present study being a retrospective study was conducted in the Dept. of Pathology ESIC Medical College\& Postgraduate Institute of Medical Sciences and Research, (ESIC MC \& PGIMSR), Rajajinagar, Bangalore. Archived cases of preceding 5 years from Jan 2012 to Nov 2016 were taken. The clinical details were retrieved from records. The study included conventional pap stained smears of ASCUS, ASC-H, LSIL, HSIL and SCC. A total of 436 cases were studied. ASC/SIL ratio was calculated. ASC component included ASC-US and ASC-H. SIL component included LSIL, HSIL and SCC.

Results: Of the total 436 cases, 226 cases were ASCUS and ASC-H and 210 cases were LSIL, HSIL and SCC. ASC/SIL ratio was obtained by dividing the sum of all ASC cases by the sum of all SIL cases. The ratio obtained was 1.1:1 which is below the upper bench mark of 3:1.

Conclusion: Monitoring the ASC/SIL ratio of a laboratory is a useful quality control measure. Bethesda system have suggested that the ratio should be less than 3 and others have suggested that lower ratios are desirable. As diagnosis of ASCUS conveys uncertainty, a low ratio decreases the uncertainty produced by laboratory and may reduce the percentage of women with negative biopsy results.

\section{Keywords: Atypical Squamous Cells, Squamous Intraepithelial Lesion, Quality Control}

\section{Introduction}

The incidence and mortality from cervical cancer is decreasing as a result of successful screening by cervical cytology. The carcinoma of cervix is the commonest malignancy reported in developing countries. About 122,844 new cases are detected every year in India and 67477 die, out of 432.2 million female population aged 15years and older. ${ }^{[1]}$ To promote optimal patient care, quality control measures for Pap smear are very essential.

Quality control forms an integral part of any laboratory system. Quality control is defined as a system for verifying and maintaining a desired level of quality in an individual test or process. ${ }^{[2]}$ The main objective of quality control is to eliminate the false negative test results. ${ }^{[3]}$ For practical purposes, quality control can be divided into internal and external quality control. Internal quality control helps in improving the performance of single laboratory, where as external quality control helps in evaluation of the performance of different laboratories and thus can help in inter-laboratory comparability ${ }^{[4]}$ The various internal quality indicators that are used in pap cytology are atypical squamous cells rate, correlation of ASC cases with results of biopsy, correlation of ASC-US cases with high risk HPV positivity rates and calculation of ASC/SIL ratio. ${ }^{[5,6] \text {. }}$

Atypical Squamous Cells (ASC) constitutes around 5\% of Papanicolaou test results and is considered as a common gynaecological cytologic abnormality. ${ }^{[7]}$ The Bethesda System for reporting Pap Cervical Cytology defines ASC as cells having cytologic changes suggestive of Squamous Intraepithelial Lesion (SIL) but lack insufficient data either qualitative or quantitative, for a definite diagnosis of SIL. ${ }^{[8]}$ It can be used as an intra-laboratory \& interlaboratory comparison tool for quality control purposes as it reflects a diagnosis of uncertainty and to ensure that this interpretation is not overused. ${ }^{[7]}$

As there is high prevalence of ASCUS interpretation, if incorrectly used affects the clinical management of patients and can result in over or under treatment of significant numbers of patients ${ }^{\left[{ }^{[9]}\right.}$ According to interim guidelines ASCUS diagnosis may be expected in about $5 \%$ of patients and greater frequency may constitute misuse of the term. ${ }^{[10]}$ 
The ratio of ASC to SIL interpretations has been adopted as a preferred measure for the frequency of cervical dysplasia in different populations. The ASC/SIL ratio has been used as a surrogate marker for the level of certainty and specificity and can be calculated for the entire laboratory or for individual cytopathologists. ${ }^{[7]}$

Bethesda authors have suggested ASC/SIL ratio of less than 3:1 for an individual or a laboratory which helps them to assess their ratio as well as the laboratory as a whole. ${ }^{[11,12]}$ This study shows how ASC/SIL ratio depends on underlying SIL prevalence and also helps to analyse the laboratory performance in reporting pap smears.

Objective of study is to assess the ratio of ASC/SIL categories and thereby evaluation of quality control in gynaecologic cytology smears.

\section{Materials and Methods}

The present study being a retrospective study was conducted in the Dept. of Pathology ESIC MC \& PGIMSR, Rajajinagar, Bangalore. Archived cases of preceding 5 years from Jan 2012 to Nov 2016 were taken. The clinical details were retrieved from records. The study included conventional pap stained smears of ASC-US, ASC-H, LSIL, HSIL and SCC.A total of 436 cases were studied. ASC/SIL ratio was calculated. ASC component included ASC-US and ASC-H. SIL component included LSIL, HSIL and SCC.

The cytologic criteria that was used for classification of the above cases was according to the Bethesda System for Reporting Cervical Cytology 2014. ${ }^{[13]}$

The following is a brief description of the criteria explained with respect to various squamous lesions as described in The Bethesda System for Reporting Cervical Cytology 2014.

ASC-US: Nuclei are approximately two and half to three times the area of the nucleus of a normal intermediate squamous cell with slightly increased ratio of nuclear to cytoplasmic area (N/C). Minimal nuclear hyperchromasia and irregularity in chromatin distribution or nuclear shape and atypical parakeratosis are characteristic.

ASC-H: Cells usually occur singly or in small fragments of less than 10 cells. Cells are the size of metaplastic cells with nuclei that are about $1 \frac{1}{2}$ to $2 \frac{1}{2}$ times larger than normal. Ratio of nuclear to cytoplasmic (N/C) area may approximate that of HSIL.

LSIL: Nuclear enlargement more than three times the area of normal intermediate nuclei results in a slightly increased $\mathrm{N} / \mathrm{C}$ ratio. Variable degrees of nuclear hyperchromasia are accompanied by variations in nuclear size, number and shape. Chromatin is uniformly distributed and coarsely granular. Nucleoli are absent or inconspicuous. Perinuclear cavitation ("koilocytosis") consisting of a sharply delineated clear perinuclear zone and a peripheral rim of densely stained cytoplasm is a characteristic feature. Alternatively, the cytoplasm may appear dense and orangeophilic (keratinized).

HSIL: Degree of nuclear enlargement is more variable than that seen in LSIL. Cytoplasmic area is decreased, leading to a marked increase in N/C ratio. Contour of nuclear membrane is irregular and frequently shows prominent indentations. Nucleoli are absent. Cytoplasm is immature, lacy and delicate.

Squamous Cell Carcinoma (SCC): Few cells are seen as singles and less commonly in aggregates with marked variation in cellular size and shape with caudate and spindle cells that contain dense orangeophilic cytoplasm. Nuclei vary markedly in size, nuclear membranes may be irregular in configuration, and numerous dense opaque nuclei are often present. Associated keratotic changes ("hyperkeratosis" or "pleomorphic parakeratosis") may be seen.

\section{Results}

Of the total 436 cases, 226 cases were ASC component which included ASCUS and ASC-H (Table 1)and 210 cases were SIL component which included LSIL, HSIL and SCC (Table 2). ASC/SIL ratio was obtained by dividing the sum of all ASC cases by the sum of all SIL cases. The ratio obtained was 1.1:1 which is below the upper bench mark of 3:1.

\section{Discussion}

The objective of quality control is to reduce the rate of false negative test results. There are various quality assurance

Table 1: Number of cases in ASC component

\begin{tabular}{|l|c|}
\hline PARAMETER & NUMBER OF CASES \\
\hline ASC-US & 100 \\
\hline ASC-H & 126 \\
\hline ASC COMPONENT & 226 \\
\hline
\end{tabular}


Table 2: Number of cases in SIL component

\begin{tabular}{|l|c|}
\hline PARAMETER & NUMBER OF CASES \\
\hline LSIL & 78 \\
\hline HSIL & 92 \\
\hline SCC & 40 \\
\hline SIL COMPONENT & 210 \\
\hline
\end{tabular}

ASC/SIL RATIO: $226 / 210=1.1: 1$

Table 3: Comparison of ASC/SIL ratios in various studies.

\begin{tabular}{|l|c|}
\hline STUDY & ASC/SIL RATIO \\
\hline Davey D D et al ${ }^{[14]}$ & 2 \\
\hline Renshaw A A et al ${ }^{[7]}{ }^{[8]}$ & 2.5 \\
\hline Nascimento A F et al ${ }^{[8]}$ & 1.9 \\
\hline Davey D D et al ${ }^{[15]}$ & 1.3 \\
\hline Renshaw A A et al ${ }^{[12]}$ & 3.2 \\
\hline Present study & 1.1 \\
\hline
\end{tabular}

monitors which may be utilized to evaluate the laboratory's utilization of ASC which are as follows: ${ }^{[5]}$

1. Correlation of ASC-US cases with high-risk HPV positivity rates; should be in the range of $40-60 \%$, or in essence that ASC-US is a 50-50 proposition between SIL (usually LSIL) and cellular changes unrelated to HPV;

2. Correlation of ASC cases with results of colposcopically directed biopsy;

3. Review of ASC cases by a second cytopathologist.

4. Calculation of ASC/SIL ratio

5. Atypical squamous cell rate; refers to ASCUS and $\mathrm{ASCH}$ categories divided by number of all gynaecologic cytology cases. ${ }^{[6]}$

However, calculation of ASC/SIL ratio is a simplest measure for assessing the quality of reports by pathologists and as well as laboratory.

In our study a total of 436 cases were studied retrospectively, out of which 226 cases belonged to ASC component and 210 cases belonged to SIL component. We obtained a ratio of 1.1:1 which is below the upper bench mark of $3: 1$.

Studies done by various authors with reference to ASC/ SIL ratio as a quality control indicator have been tabulated below (Table 3).

Our study showed concordance results with Davey D D et al. ${ }^{[9]}$ who obtained a ratio of 1.3 .

A study conducted by Juskevicius et al. ${ }^{[8]}$ showed that by providing ASC/SIL ratio of individual cytopathologists (CPs) in a confidential communication was a useful quality control measure as it helps in analysing their performance. The frequency of ASCUS interpretation in a general population should not be greater than $5 \%$ to avoid the misuse of the term. As ASC/SIL ratio are less dependent on patient population, they increase with more number of high risk patients in a laboratory. ${ }^{[16]}$

Although ASC/SIL ratio is a measure of a cytopathologist's uncertainty, is certainly not a measure of cytopathologists diagnostic accuracy. ${ }^{[8]}$ Overall performance evaluation stake not just ASC/SIL ratio but also other parameters such as biopsy and HPV correlation data. ${ }^{[8]} \mathrm{As}$ monitoring the ASC/SIL ratio is a useful quality control measure, allows individual CPs to assess their ratio as well as the laboratory as a whole and against the 3:1 benchmark. Bethesda system suggests that the ratio should be less than 3 and the lower ratios are more desirable as low ratio decreases the uncertainty produced by laboratory and may help to reduce the percentage of women with negative biopsy results. ${ }^{[12]}$

A study conducted by Renshawet al. in his study concludes as follows: "A laboratory depends on its cytotechnologists to maintain adequate sensitivity and on its cytopathologists to maintain adequate specificity". ${ }^{[16]}$

\section{Conclusion}

ASC/SIL ratio serves as a good surrogate marker for screening the sensitivity of cytopathologists. Confidential feedback of ASC/SIL ratio of individual cytopathologists helps in diminution of their ratio if it exceeds the upper bench mark. For cytopathologistswhose ratio correlates with the established bench mark, acts as a positive reinforcement and helps to maintain a stable ratio. 


\section{References}

1. Sreedevi A, Javed R, Dinesh A. Epidemiology of cervical cancer with special focus on India. Int J Women's Health. 2015;7:405-414.

2. Krieger PA, McGoogan E, Voojis GP, Amma NS, CochandPriollet B et al. Quality Assurance / Control issues. ActaCytologica. 1998;42:133-140.

3. Tobias AHG, Amarlal RG, Diniz EM, Carneiro CM.Quality Indicators of Cervical Cytopathology Tests in the Public Service in Minas Gerais, Brazil. Rev Bras GinecObst.2016;38:65-70.

4. Azara CZS, Manrique EJC, Alves de Souza NL, Rodrigues AR, Nascimento Tavares SBD. Quality Control of Cervical Cytopathology: Interlaboratory Variability. Acta Cytologica.2013;57:585-590.

5. Bibbo M, Wilbur DC ; Comprehensive Cytopathology. 3 rd ed. Saunders: Elsevier; 2008.

6. Auger M. Selection of Monitoring Parameters for Gynecologic Cytology_Beacons of Light for Quality Assurance. Cancer Cytopathology 2014; DOI: 10.1002/ cncy. 21349

7. Renshaw AA, Deschênes M, Auger M. ASC/SIL Ratio for Cytotechnologists: A Surrogate Marker of Screening Sensitivity. Am J ClinPathol. 2009;131:776-81.

8. Nascimento AF, Cibas ES. The ASC/SIL ratio for Cytopathologists as a Quality Control Measure: A Followup Study. Am J ClinPathol. 2007;128:653-56.

9. Ko V, Nanji S, Tambouret R H, Wilbur D C. Testing for $\mathrm{HPV}$ as an Objective Measure for Quality Assurance in Gynecologic Cytology Positive Rates in Equivocal and Abnormal Spec imens and Comparison With the ASCUS to SIL Ratio. Cancer Cytopathology 2007;111(2):67-73.
10. Davey DD, Naryshkin S, Nielsen ML, Kline TS. Atypical squamous cells of undetermined significance: interlaboratory comparison and quality assurance monitors. DiagnCytopathol. 1994;11:390-96.

11. Juskevicius R, Zou KH, Cibas ES. An analysis of factors that influence the ASCUS/SIL ratio of pathologists.Am $J$ ClinPathol. 2001;116:331-35.

12. Renshaw AA, Genest DR, Cibas ES. Should Atypical Squamous Cells of Undetermined Significance (ASCUS) Be Subcategorized?: Accuracy Analysis of Papanicolaou Smears Using Receiver Operating Characteristic Curves and Implications for the ASCUS/Squamous Intraepithelial Lesion Ratio. Am J ClinPathol. 2001;116:692-95.

13. Nayar R, Wilbur DC. The Bethesda System for Reporting Cervical Cytology. Definitions, Criteria, and Explanatory Notes. 3 rd ed. New York: Springer; 2015.

14. Davey DD, Woodhouse S, Styer P, et al. Atypical epithelial cells and specimen adequacy: current laboratory practices of participants in the College of American Pathologists Interlaboratory Comparison Program in Cervicovaginal Cytology. Arch Pathol Lab Med. 2000;124:203-11.

15. Davey DD, Nielsen ML, Naryshkin S, et al. Atypical squamous cells of undetermined significance: current laboratory practices of participants in the College of American Pathologists Interlaboratory Comparison Program in Cervicovaginal Cytology. Arch Pathol Lab Med. 1996;120:440-44.

16. Catteau X,Simon P, Noel J C. Evaluation of the Oncogenic Human Papillomavirus DNA Test with Liquid-Based Cytology in Primary Cervical Cancer Screening and the Importance of the ASC/SIL Ratio: A Belgian Study. ISRN Obstetrics and Gynecology 2014; http://dx.doi. org/10.1155/2014/536495.

*Corresponding author:

Dr. Hemalatha. J, Department of Pathology, ESIC Medical College \& Postgraduate Institute of Medical Sciences and Research, Rajaji Nagar, Bangalore, Karnataka (India) - 560010

Phone: +91 9740614607

Email: drhemalathaj@gmail.com

Date of Submission : 21.03.2017

Financial or other Competing Interests: None. 